\title{
A Mobile User Interface for Business Knowledge Management (BKM)
}

\author{
Danco Davcev, Marjan Arsic, and Dalibor Ilievski \\ University "Ss Cyril and Methodius", Skopje, Macedonia, \\ Faculty of Electrical Engineering and Information Technologies \\ etfdav@feit.ukim.edu.mk, a_marjan@t-home.mk, i_dalibor@yahoo.com
}

\begin{abstract}
In this paper, we introduce an adaptable mobile interface for efficient business knowledge management (BKM). The user (client) via the Helpdesk operator can post a request for consultation to the business Experts and/or web services. The goal is to make business knowledge available on demand at any time and any place. This approach gives faster business problem solutions, more productive expert and/or web services, and high availability of the business knowledge.
\end{abstract}

Keywords: Adaptable mobile interface, Web services, XML Agents, Fuzzy logic, Pocket PC.

\section{Introduction}

Exchange of knowledge and consultation process among customers and available expert authority via helpdesk operators are very important aspects of quick problem solution using the mobile devices.

The goal of the mobile technology is to make information instantly available to customers. The new business processes can benefit a lot by using mobile connection between customers and experts. The exchange of the various content types of media data (text, drawing, and sound) will enhance the efficiency of consultations between the customer and the expert advisor. It gives benefits in reducing of costs, avoiding future problems or possible claims, saving time, increasing profit and customer satisfaction.

There are many desktop applications that support multimedia communication among participants. Communication among several participants that includes multimedia transfer is at the beginning of the development and utilization in the sphere of mobile handheld devices. Hence, new demands are imposed to these devices: greater processing power to support real time multimedia transfer (video stream, voice stream, and file exchange), greater memory space. Modern feature rich applications demand space on the screen (interface) for displaying all available features, which could be an issue when handheld devices are used.

A great consideration should be given to the readability and utilization of small mobile user interfaces. In this paper, we present our approach in creating an adaptable mobile interface for demanding and exchanging of various content types and knowledge on mobile devices. The interface adapts the dimensions and visibility of the user 
controls according to the user's preferences and utilization of the controls and also, the current usage of the specific media contents within the business process. Our adaptable mobile interface is managed by fuzzy logic and many XML based agents.

The related work in the second section gives some comments to similar work. The architecture of our business knowledge management (BKM) system is elaborated in the third section, which is the main contribution of this paper. The fourth section describes the design of the system. Implementation and Evaluation of system are presented in fifth section. Finally, in the sixth section we conclude the paper.

\section{Related Work}

The agent-based approach that uses fuzzy logic to determine importance of certain information is elaborated in [1]. The agents described in this paper are based on access to the Web. We use similar approach to determine importance of user interface features in regards to user preferences and media contents used in current session. However, in our approach, wireless communication between handheld devices is used.

In [2] an agent based adaptive user interface control for desktop applications is presented. Although we share similar ideas, our approach is multi-agent based adaptive interface which uses fuzzy logic for handheld devices.

In [3], collaborative applications can be built using two types of agent collaborations, agent communications through XACL and agent visiting. Agents are represented as XML entities, not programming language entities. Secondly, agent hosting services are implemented as web services with published WSDL, not programs in certain programming languages with published APIs. Thirdly, although XML agents' behaviors have to be coded in some supported programming languages, the interactions between agents and hosts in agents' behavior code are through invoking local hosts' web services, which is neutral to programming languages and host operating systems.

Although we also use XML entities as agents and we share similar ideas of using web services (when appropriate), in our approach we use more flexible protocol for communication among agents which is based on fuzzy-based knowledge.

In [4], an XML-based multiagent recommender system for supporting online recruitment services is proposed. Although the main purpose of this system is to provide Online Recruitment Services, it is also agent and XML based and as a consequence, it can easily cooperate with company information systems. The so called ontology of their user agent stores the profile of a given user concerning the job search. It is different from our system because we plan to build a general purpose knowledge management system (not only for recruitment services). For the time being, our objective is to have a self - adaptive system from the point of view of an efficient communication between user and the advisor according to the user's preferences and the current possibilities of the communication system.

In [5] a feasible framework that combines agent mobility and intelligence for consumer-oriented e-business applications is proposed. This framework complements the current Web-based systems by adding the wireless channel of mobile agents. In our work the mobile agents use an adaptive communication protocol based on fuzzy logic. In addition, our framework includes web services. 


\section{BKM System Architecture}

There are three kinds of users like customers, helpdesk operator and experts. Helpdesk operator is the link between experts and customers.

The goal of the helpdesk operator is to response any kind of requests on demand by the customers in appropriate formats (text, draw, VoIP) and to send the complex requests to the experts and/or web services which should be resolved by them. Client applications are implemented on Pocket PCs.

There is only one helpdesk operator and many experts in one consultation session.

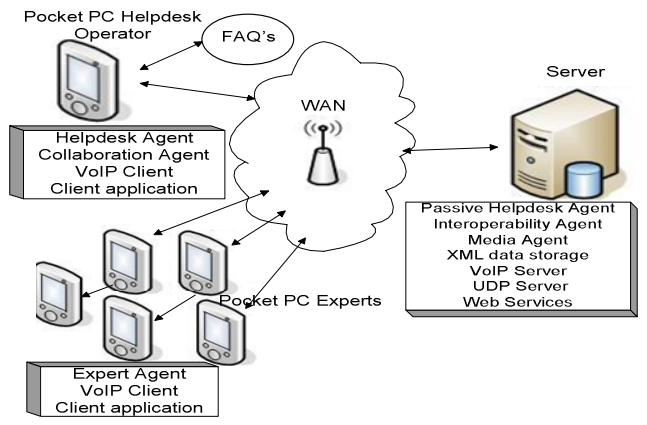

Fig. 1. Architecture of BKM system

The network communication is realized in a way that clients send UDP datagrams to the server, and the server resends the UDP datagrams, according to the contents of the received message.

The communication protocol between the agents and the information flow rely on fuzzy logic. In this case, the fuzzy logic is used for enabling or disabling (adaptation) some controls depending on the quality of the service (QoS), i.e. the signal strength. So, if the signal strength is good, the features of chat, draw and VoIP will be enabled. However, for medium signal the VoIP feature will be disabled. For bad signal, beside the VoIP, the draw feature should be also disabled, and the only active communication feature will be chat.

Streams are used for the voice transfer. Desktop based clients (for both helpdesk operator and experts) can be also connected to the server.

The Web services are used to find the solution of the problem using the wireless Internet access. If the Web services are unable to find the solution, the Experts are activated. Some types of Web services are used for the responses. These Web services support and convert various types of media data.

The experts interface has controls for chat, draw, VoIP, file upload/download, authority control (VoIP channel).

The helpdesk operator can give the control over the interface (or by giving the speaking possibility) and reclaim the control later. Helpdesk operator has the role of a moderator in the consultation process.

Figure 1 shows the architecture of the BKM system. Client applications reside on pocket PCs and they have three main parts: Helpdesk agent, which manages operator 
interaction within the application and communicates with interoperability agent. VoIP client serves for the voice transfer, and the client application represents all the features mentioned above (chat, draw, voice, file up/download, communication among clients). Expert Agent receives and sends the resolved requests using various file types (like text, image, and voice). Collaboration Agent calculates the cost of the corresponding expert's service. The communication is realized through wireless area networks connected to the Internet.

The server contains Interoperability Agent which redirects the complex request to the Experts and/or Web services and exchanges reformatted media types; Passive helpdesk Agent calculates the helpdesk interface; Media Agent monitors the media contents (text, image or voice); Experts and helpdesk preferences are stored in XML data storage; VoIP Server is responsible for voice transfer and UDP server application manages the login, chat, draw and control. For the purpose of saving the memory and processing power of the clients handheld devices, Passive Helpdesk Agents and Media Agent reside on the server side. The agents are XML based.

\section{BKM System Design}

In this paper, we describe our approach in development of a BKM system by using multi XML agents and fuzzy logic. Helpdesk agent resides on the client side, and monitors the helpdesk operator's interaction by using the features of the application and communicates with interoperability agent (e.g. sends the request to the available competent experts and/or web services, receives the response in corresponding format and regulates the customers billing). The triggers that demand helpdesk agent's actions are: drawing, sending chat messages, and communicating using VoIP. This agent is named Helpdesk because it helps the customers to simply resolve their requests. At the start of the communication session, helpdesk agent communicates with the Passive helpdesk agent and interoperability agent that reside on the server. The Passive agent demands the information from the Media agent about the type of the files to be used for the particular communication session. This agent is named passive helpdesk since it waits to be invoked; it recalculates the interface structure and sends the data to the helpdesk agent which finally draws the interface. The Expert Agent receive the requests in some format (text, file, voice) from the interoperability agent, concerns about the solution of the requests, sends the solutions to interoperability agent, modifies the expert's profile, checks the QoS and billing status.

The main tasks of the interoperability agent are: receiving the requests from helpdesk agent and forwarding them to the expert agents and/or web services. Also it exchanges reformatted data files with helpdesk agent. Media agent monitors the folder with the data files for the communication session, and sends this information to the Passive helpdesk agent. The Passive helpdesk agent recalculates the helpdesk interface according to the information from the Media Agent, the history of interface affinities (chat_aff, draw_aff, sound_aff, QoS) for the particular user and the helpdesk agent's message about the use of the features. Then, it sends the corresponding information (for the type of the helpdesk interface) to the Helpdesk agent which adapts the helpdesk interface according to this information. The collaboration Agent calculates the time session for realization of the expert task and the cost of the service. 
Helpdesk and Expert profiles with personal information about the qualification and the knowledge for all of them are stored in the XML database. Additionally a history of affinities is stored for the helpdesk operator and the experts. According to the history, Passive helpdesk agent adapts the helpdesk interface on the beginning of the communication session.

There are currently three predefined user interfaces, which can be preloaded on the client's device, according to his preferences and the media contents for the current communication session. The dimensions and the positions of the controls would be stored on the helpdesk's affinity table on the server. One kind of an interface displays chat on the larger part of the screen, the second interface has larger drawing view on the screen, and the third one has equal space for the chat and the drawing space. VoIP feature doesn't occupy much space on the screen, so the buttons for voice are always displayed and they will be active if the Signal Strength is satisfied, i.e. QoS is strong.

Since there is no universal conclusion for making decisions of which user interface should be preloaded, fuzzy logic approach is used to model that kind of the imprecise information.

The interaction made by the helpdesk operator using the features of the application (chat, draw and voice feature), is represented as a vector (chat_aff, draw_aff, sound_aff). A linguistic variable named RESULT_PREFERENCE is introduced, and it accepts values from the set of terms \{increase_chat, increase_draw, the_same\}. This variable represents the visualization of the helpdesk interface, produced by (1) the helpdesk operator interaction with the interface; $(2)$ the contents of media folder and (3) the quality of service of the signal strength that decides more accurately which interface to be preloaded.

The class diagram for WiFi signal strength is shown on Figure 2. Two classes are developed, WiFiPeek and SignalStrength. The WifiPeek class implements all the WiFi query related elements. The class uses the NDIS User mode I/O driver (NDISUIO) to perform Access Point (AP) scanning.

The GetAdapters function can be used to query names of network adapters. It calls the built-in NDIS (not NDISUIO) driver. The function fills a buffer with adapter names separated by commas. The RefreshBSSIDs function requests that the driver initiate an AP survey. It takes one parameter: an adapter name. The GetBBSIDs function returns the list of available stations, i.e. peers and Access Points. The function

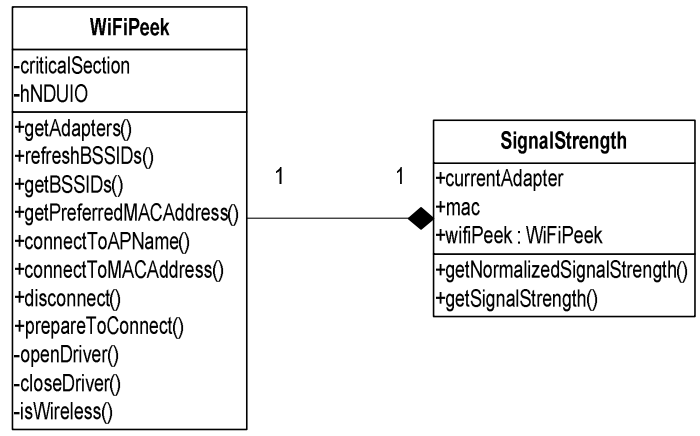

Fig. 2. WiFi signal strength - class diagram 
getPreferredMACAddress returns the MAC address of the connected (associated) Access Point.

In order to calculate the user interface visual features, the following fuzzy variables are defined for this expert system: U_A (user affinity) which presents the most used feature by the user, i.e. it has the greatest affinity for the feature; M_P (media profile) presents the profile of the interface that should be used according to the media contents; QoS (Quality of Service) presents the strength of the wireless signal. Output variable named I (action for interface adaptation) presents the necessary interface to be preloaded onto the user's device screen, according to the input variables.

The linguistic variable Quality of Service (QoS) accepts values from the set of terms $\{$ Strong, Medium, Weak $\}$.

The linguistic variable U_A accepts values from the set of terms \{Chat, Draw, VoIP and it has normalized values of utilization between 0 and $1(0 \%$ to $100 \%)$. It is equivalent to the number of times of usage of the features chat, draw or VoIP respectively.

The linguistic variable M_P accepts values from the set of terms \{Text, Drawing, Sound . It has normalized values of utilization between 0 and $1(0 \%$ to $100 \%)$, which is equivalent to the number of text, drawing and sound files in the media profile respectively.

In the table 1 is presented the simulation for fuzzy linguistic values for U_A, M_P and QoS and the value of I is calculated, based on the fuzzy rules. The action needed to adapt the interface is described with the following XML code like the example 1 of the Table 1.

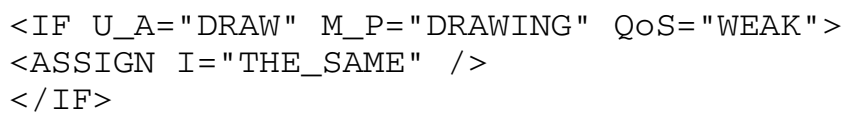

Table 1. Some examples for adapting interface using fuzzy linguistic variables

\begin{tabular}{|c|c|c|c|c|c|c|}
\hline Examples & 1 & 2 & 3 & 4 & 5 & 6 \\
\hline User Affinity (U_A) & \multicolumn{6}{|c|}{ Utilization \% } \\
\hline CHAT & $\mathbf{3 8 , 0 0 \%}$ & $57,69 \%$ & $57,69 \%$ & $11,90 \%$ & $31,58 \%$ & $41,86 \%$ \\
\hline DRAW & $46,00 \%$ & $23,08 \%$ & $23,08 \%$ & $28,57 \%$ & $31,58 \%$ & $23,26 \%$ \\
\hline VOIP & $16,00 \%$ & $19,23 \%$ & $19,23 \%$ & $59,52 \%$ & $36,84 \%$ & $34,88 \%$ \\
\hline Media Profile (M_P) & \multicolumn{6}{|c|}{ Utilization $\%$} \\
\hline TEXT & $26,67 \%$ & $\mathbf{3 0 , 3 0 \%}$ & $\mathbf{3 0 , 3 0 \%}$ & $39,47 \%$ & $29,09 \%$ & $76,19 \%$ \\
\hline DRAWING & $50,00 \%$ & $54,55 \%$ & $54,55 \%$ & $34,21 \%$ & $30,91 \%$ & $14,29 \%$ \\
\hline SOUND & $23,33 \%$ & $15,15 \%$ & $15,15 \%$ & $26,32 \%$ & $40,00 \%$ & $9,52 \%$ \\
\hline Q.0S & \multicolumn{6}{|c|}{ Signal Strength $\%$} \\
\hline STRONG & $0,00 \%$ & $0,00 \%$ & $10,00 \%$ & $0,00 \%$ & $100,00 \%$ & $100,00 \%$ \\
\hline MEDIUM & $10,00 \%$ & $25,00 \%$ & $90,00 \%$ & \begin{tabular}{|l|}
$100,00 \%$ \\
\end{tabular} & $0,00 \%$ & $0,00 \%$ \\
\hline WEAK & $90,00 \%$ & $75,00 \%$ & $0,00 \%$ & $0,00 \%$ & $0,00 \%$ & $0,00 \%$ \\
\hline $\begin{array}{l}\text { Action for Interface } \\
\text { Adaptation (l) }\end{array}$ & \multicolumn{6}{|c|}{ Fuzzy Value $\%$} \\
\hline INCREASE_CHAT & $38,00 \%$ & $54,55 \%$ & $\mathbf{3 0 , 3 0} \%$ & $39,47 \%$ & $31,58 \%$ & $41,86 \%$ \\
\hline INCREASE_DRAW & $10,00 \%$ & $23,08 \%$ & $23,08 \%$ & $34,21 \%$ & $31,58 \%$ & $14,29 \%$ \\
\hline THE_SAME & $48,00 \%$ & $25,00 \%$ & $54,55 \%$ & $28,57 \%$ & $36,84 \%$ & $23,26 \%$ \\
\hline
\end{tabular}


In the first example from the Table 1, the draw affinity is more utilized than chat and VoIP and the media profile has more drawing objects than text or sound. It is expected to be increased the drawing interface, but because the signal is weak and the drawing features are not supported on such signal, the interface stay unchanged (the_same). The total numbers of fuzzy rules are 27, the combination of the values of all linguistic variables. The next examples are similar like the first one.

The interaction among agents is shown on the Figure 3.

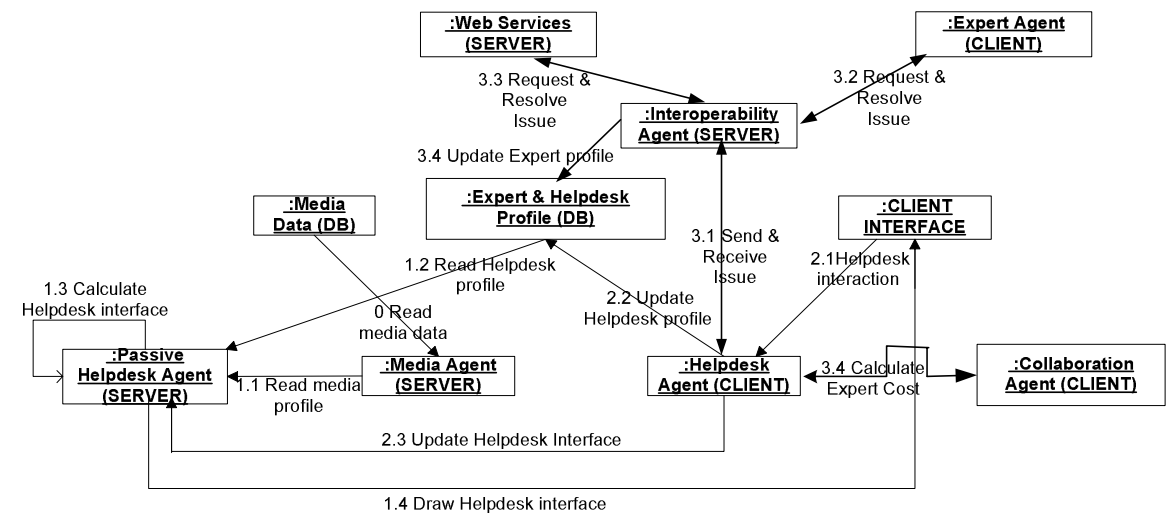

Fig. 3. Agents' collaboration diagram

\section{BKM System Implementation and Evaluation}

BKM system is developed in C++ Visual Studio .NET 2008 development framework with using of the MFC (Microsoft Foundation Classes) library. Operating systems used on the pocket PCs are Windows CE, Windows Mobile. For BKM system testing is used WLAN Internet provided from T-Home, Macedonia. The clients which reside on desktop PCs and the server use the standard .NET Framework. Operating system for desktop machines on which the application is practically deployed, is Windows XP SP2. The interface adaptation is realized on different screen resolutions.

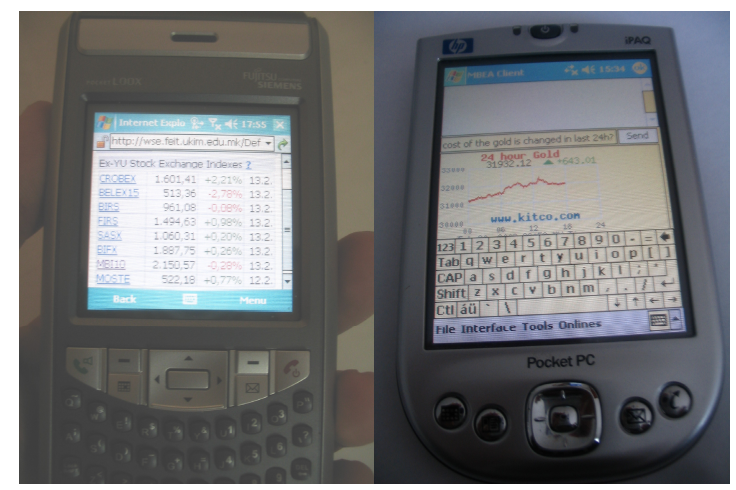

Fig. 4. Two instances of the BKM system 
The two instances of BKM interface are shown on Figure 4 for different screen sizes of Pocket PC-s.

The initial feedback of BKM system was given by 30 colleagues in ICT. They were asked to use the system for stock exchange analysis in last week. General opinion among participants for the interface usability, functionality and visibility is average $(71,3 \%)$. Interface is functional and suggestions for interface rearrangment are mostly done according to the user needs.

The questions that examine interface usability, functionality, visibility and provide information for future upgrades are:

1. Are you satisfied with the way of adaptive presentation?

2. Can you easily select the parameters of BKM system?

3. Were the results of the BKM system solutions are clearly displayed?

4. How much the BKM system helped in the business process?

5. Does the BKM system satisfy the quality of the service?

The questions are answered with "Yes", "No" or "I Don't Know". The answers are presented in the chart on Figure 5.

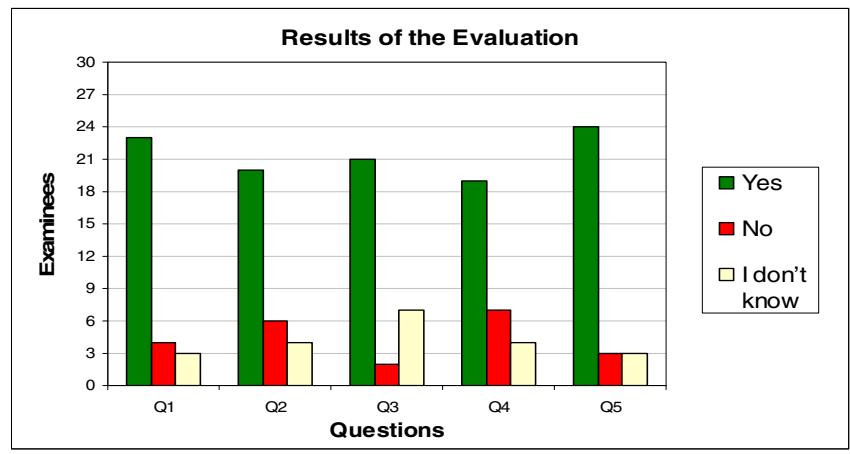

Fig. 5. Results of the examinees' opinion about the BKM system

\section{Conclusion}

This paper presents our approach that uses XML agents and fuzzy logic in order to achieve adaptive interface for a BKM system. We have developed such mobile system which adapts the features of the interface according to the contents for the specific session. Fuzzy logic approach is used for the communication protocol between XML agents and for interface adaptation. It can be also used to dynamically reconfigure the interface according to the preferences and the type of request. In this way, we proposed an adaptable interface for our BKM system to all mobile device users. General opinion among participants for the BKM system usability is positive. 


\section{References}

1. Camacho, D., Hernandez, C., Molina, J.M.: Information Classification Using Fuzzy Knowledge Based Agents. In: IEEE International Conference on Systems, Man, and Cybernetics, pp. 2575-2580 (2001)

2. Lu, L., Kitagata, G., Suganuma, T., Kinoshita, T.: Adaptive user interface for multimedia communication system based on multiagent. In: Proc. of the 17th International Conference on Advanced Information Networking and Applications, pp. 53-58. IEEE Computer Society, Los Alamitos (2003)

3. Du, W., Li, H.: XML Agents Technology for Building Collaborative Applications. In: Collaborative Technologies and Systems, CTS International Symposium, pp. 289-297 (2006)

4. Meo, P.D., Quattrone, G., Terracina, G., Ursino, D.: An XML-Based Multiagent System for Supporting Online Recruitment Services. IEEE Transactions on Systems, Man and Cybernetics, Part A 37(4), 464-480 (2007)

5. Weng, Z., Tran, T.: An Intelligent Agent-Based Framework for Mobile Business. In: International Conference on Management of Mobile Business, ICMB 2007, p. 30 (2007) 